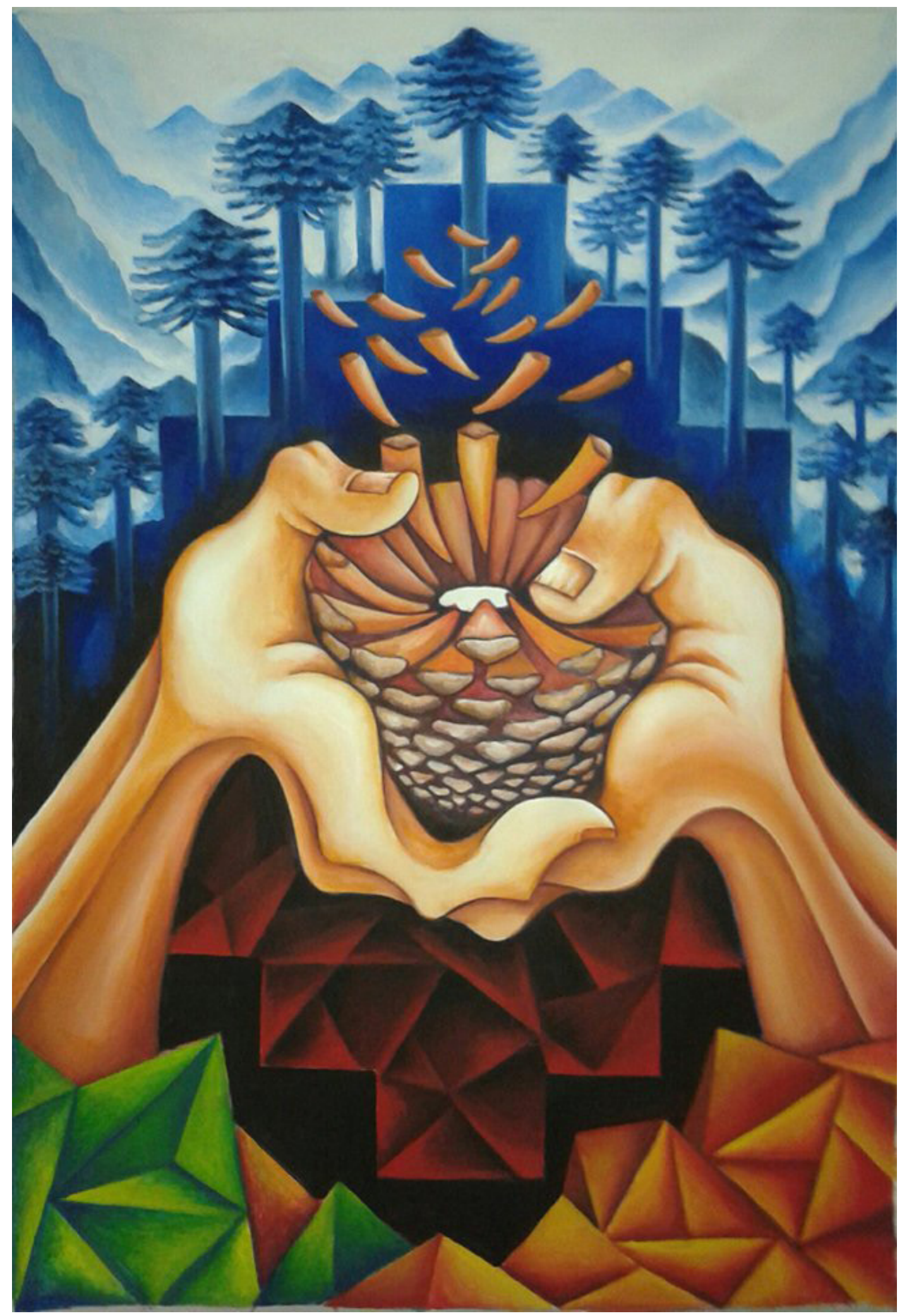

Artista: Heri Tapia

Antaño,

antaño fue cuando sobre los indios se abrió como una rosa de madera el colosal puñado de tu puño, Y dejó sobre la mojada tierra Los Piñones:

Harina, pan silvestre del indomable Arauco...

P. Neruda... 


\title{
Facebook y Youtube: tecnologías del aprendizaje y el conocimiento para el fortalecimiento de la competencia comunicativa en la clase de lengua castellana
}

\author{
FACEBOOK AND YOUTUBE: LEARNING AND KNOWLEDGE TECHNOLOGIES FOR STRENGTHENING \\ COMMUNICATIVE COMPETENCE IN THE SPANISH LANGUAGE CLASS \\ FACEBOOK E YOUTUBE: TECNOLOGIAS DA APRENDIZAGEM E O CONHECIMENTO PARA O \\ FORTALECIMENTO DA COMPETÊNCIA COMUNICATIVA NA AULA DE LÍNGUA CASTELHANA
}

\author{
Leydy Garay Álvarez 1 / leydygaray@hotmail.com \\ Luisa Fernanda Acuña Beltrán²/ lacuna@idep.edu.co
}

\section{Resumen}

Este artículo es el resultado del trabajo de investigación Facebook y YouTube: tecnologías del aprendizaje y el conocimiento para el fortalecimiento de la competencia comunicativa en la clase de lengua castellana, realizado para optar al título de Magíster en Pedagogía en la Universidad de la Sabana. La investigación se realizó con el propósito de determinar de qué forma el uso de Facebook y YouTube fortalece la competencia comunicativa de los estudiantes del ciclo 5 (grados décimo y undécimo) de la jornada tarde del Colegio Saludcoop Norte I.E.D., en la clase de lengua castellana.

\section{Abstract}

This article presents the result of the research entitled Facebook and YouTube: Learning and knowledge technologies for strengthening communicative competence in the Spanish language class, in order to obtain the Master's Degree in Pedagogy at the Universidad de la Sabana. The research aimed to determine how the use of Facebook and YouTube reinforce the fifth cycle studnets' (10th and 11th graders) communicative competence in the afternoon session at the Colegio Saludcoop Norte (District Educational Institution), in the Spanish language class.

\section{Resumo}

Este artigo é produto do trabalho de pesquisa Facebook e YouTube: tecnologias da aprendizagem e o conhecimento para of fortalecimento da competência comunicativa na aula de língua castelhana, realizado para obtenção do título de Mestre em Pedagogia na Universidade de la Sabana. A pesquisa foi feita visando determinar como o uso do Facebook e Youtube fortalece a competência comunicativa dos estudantes do ciclo 5 (Décimo e undécimo ano) no horário da tarde do Colégio Saludcoop Norte I.E.D (Instituição de Educação Distrital), na aula de língua castelhana.

Fecha de recepción: 08 de agosto de 2015 / Fecha de aprobación: 10 de septiembre de 2015

1 Licenciada en Lengua castellana. Colegio Saludcoop Norte IED, Bogotá D.C. Magíster en Pedagogía. Magíster en Formación de profesores de español como lengua extranjera.

2 Licenciada en Psicología y Pedagogía. Magíster en Estructuras y procesos del aprendizaje. Docente e investigadora Universidad de La Sabana - Maestría en Pedagogía. Investigadora del Instituto para la investigación educativa y el desarrollo pedagógico - IDEP.
Palabras clave

Competencia comunicativa, Facebook, YouTube, TIC, TAC

Key words

Communicative competence, Facebook, YouTube, TIC, TAC

Palavras chave

Competência comunicativa, Facebook, YouTube, TIC, TAC 


\section{Introducción}

La competencia comunicativa (Hymes, 1996), definida como el saber hacer cosas con el idioma en situaciones reales de comunicación, es una de las competencias más importantes para fortalecer desde la enseñanza del español como lengua propia, dado que exige que el alumno antes de escribir o producir cualquier tipo de texto tenga en cuenta, no solo las características propias del texto, sino su papel como escritor (hablante), a quién dirigirá su texto (oyentes), la situación de comunicación y la intención comunicativa del mismo.

El fortalecimiento de esta competencia en la sociedad de las tecnologías de la información y la comunicación -TIC- propone retos tanto para el docente de lengua castellana como para los estudiantes, en la medida en que requiere de ellos pasar de las tecnologías de la información y la comunicación -TIC- a las tecnologías del aprendizaje y el conocimiento -TAC.

En este sentido, el presente artículo da cuenta de los resultados de una investigación que le apuntó al uso de la web social en la clase de lengua castellana para el fortalecimiento de la competencia comunicativa, a través de la producción de textos escritos en los que se evidencie comprensión de los elementos de situación comunicativa: hablantes reales, contexto, intención, información, creencias y visiones de mundo compartidas.

Para el dominio de las reglas de uso del idioma, los estudiantes debían comprender cómo funcionaba la comunicación real, así como cuáles eran los elementos involucrados en dicho proceso. Según la teoría pragmática (Escandell, 1996), cuando se usa la lengua intervienen, además de los elementos propios del mensaje, el hablante o emisor, el oyente o destinatario, la intención comunicativa, el contexto verbal y la situación o conocimiento de mundo. Estos elementos permiten determinar cómo funciona la lengua en la comunicación.

En consecuencia, al enseñarle a los estudiantes a hacer cosas con el idioma fue necesario que ellos determinaran de manera clara que en todo proceso de comunicación son importantes los siguientes elementos: la intención de quien escribe o prepara el mensaje; las características del tipo de mensaje; el medio a través del cual se comunica ese mensaje para que sea efectivo; las características de quien o quienes recibirán dicho mensaje y la reacción o respuesta que se espera después de comunicado el mensaje. Esto es lo que desde la pragmática se conoce como las reglas de uso de una lengua; las cuales se dan de manera natural porque resultan de la experiencia social de cada individuo. Dicho de otra forma:

El uso de la lengua consiste en su intervención entre dicho estímulo y dicha respuesta y puede considerarse una reacción sustitutiva del hablante que, actuando como estímulo sustitutivo sobre el oyente, permite que este último manifieste la reacción de comportamiento deseada. (Bertuccelli, 1996, p. 82)

El estímulo resulta de la intención del hablante de comunicar un mensaje. Este mensaje debe adecuarse a su intención, de manera que logre sus objetivos comunicativos. Además, debe determinar si el canal escogido para comunicar su mensaje es el adecuado, de modo que obtenga la respuesta deseada y no haya confusiones comunicativas; es así como la lengua interviene como medio para lograr intenciones de los hablantes fruto de sus necesidades y motivaciones. Por tal razón, es clara la importancia de fijar cuáles son las motivaciones de los hablantes para comunicar su mensaje, qué los mueve a expresar su idea de una manera y no de otra, o cuál es la forma más adecuada de usar la lengua para obtener sus propósitos.

\section{El proceso realizado}

Para el desarrollo del estudio se tuvieron dos ideas como punto de partida: 1. los alumnos del ciclo 5 utilizan las redes sociales y la Internet de manera indiscriminada y 2. Es importante que la escuela ejerza un papel mediador entre los estudiantes, Internet y las redes sociales. En consecuencia, se planteó la necesidad de hacer de las clases espacios verdaderamente comunicativos para los estudiantes, haciendo uso pedagógico de herramientas TIC utilizadas en su vida cotidiana.

La investigación se sitúa en los usos reales de la comunicación que hacen los alumnos, así como en el tema de las TIC y los medios de comunicación. En este sentido, se apostó a utilizar las TIC para crear un ambiente 
de aprendizaje en el cual los estudiantes, más allá de analizar información, produjeran textos y fortalecieran su competencia comunicativa. El concepto de competencia comunicativa está ligado a la teoría pragmática e involucra aspectos como contexto e intención comunicativa para el análisis de la comunicación y bien puede abordarse desde una o varias de las habilidades comunicativas (hablar, escuchar, leer y escribir).

Así las cosas, el proceso de investigación se centró en utilizar un espacio en Internet, conocido y usado por los alumnos para "Ilenarlo" de textos propios. No fue una tarea fácil, por cuanto implicaba la realización de varios procesos complejos: el análisis, la interpretación y la comprensión de las temáticas; la escritura de un texto con características particulares; la determinación de las estrategias más eficaces para conseguir los fines comunicativos, entre otras. Además, se tuvieron en cuenta variables externas que afectan o inciden en el proceso tales como: dificultades relacionales entre los estudiantes, inconvenientes de tipo técnico, falta de compromiso de algunos estudiantes frente al trabajo, etc.

La respuesta estaba en establecer desde la práctica diaria ese deseo de hacer comunicativa la enseñanza del español con los estudiantes, aplicando fundamentos teóricos que permitirían centrar el estudio en un problema concreto. Pero surgieron cuestionamientos acerca de: ¿las TIC para qué? ¿Cuáles TIC? ¿En cuánto tiempo? ¿Cuándo? ¿Con quiénes? Las posibles respuestas a estos y otros interrogantes comenzaron a generar reflexiones como: las TIC para crear espacios de interacción que permitieran fortalecer la competencia comunicativa; redes sociales y programas que involucraran audio y video; diseñar una unidad didáctica; en las clases, procurar un espacio realmente comunicativo, en el que no solo la profesora se comunica, sino, y en mayor medida, los estudiantes de todos los cursos con los que desarrollaba la clase de lengua castellana. En este sentido, se abrió la posibilidad de aplicar una teoría con cinco grupos de estudiantes con características de aprendizaje diferentes. El proceso inició cuando los alumnos estaban en grados noveno y décimo; y se concluyó con alumnos de décimo y undécimo. Tres grupos de décimo y dos de undécimo. Sin embargo, pese a las particularidades de los alumnos, las dos unidades didácticas propuestas se realizaron en su totalidad.
En consecuencia, se diseñó e implementó una unidad didáctica denominada: De la clase de literatura a la red, cuya ruta de trabajo fue la siguiente: explicación de la teoría y aclaración de dudas al respecto (la docente); escritura de los textos literarios (los estudiantes); lectura en voz alta de los textos literarios escritos (los estudiantes); reescritura y corrección de los textos (estudiantes y docente); realización de una imagen acorde con el texto literario (los estudiantes); digitalización de la imagen (la docente); grabación de la voz en el programa Audacity (los estudiantes); grabación de la presentación en video con la cámara del computador portátil, usando YouCam (los estudiantes) y edición de los textos con el programa Movie maker (los estudiantes).

El resultado de esta unidad fue la creación de textos en formato MP4, los cuales fueron compartidos de manera pública a través de las redes Facebook y YouTube para ser observados y evaluados por los demás compañeros, utilizando una rúbrica con aspectos referidos a elementos de coherencia y cohesión textual, dominio de la temática, derechos de autor, entre otros.

\section{Resultados y hallazgos}

En cuanto a los procesos alcanzados a través de la investigación con estos cinco grupos tenemos:

1. Se propuso trabajo cooperativo (formar parejas) para que desarrollaran las actividades. Ellos decidieron, de acuerdo con sus habilidades, gustos y preferencias qué harían a lo largo de la unidad didáctica.

2. En los grupos donde había un número superior de niñas con respecto al de niños, el trabajo se centraba más en la imagen que querían transmitir.

3. Los grupos con menor rendimiento académico disfrutaban más del espacio de lectura en voz alta; sobre todo en los casos en que consideraban que sus textos habían quedado muy bien, e incluso consideraban que podían divertir a sus compañeros con lo que habían escrito.

4. Los alumnos con mayor motivación para usar recursos TIC (computadores, programas de audio y video, cámara) ofrecían su ayuda a los compañeros que tenían dudas en estos aspectos. 
5. Integrar el proceso de escritura con la creación de una imagen permitió a quienes tienen dificultades para la escritura o a quienes no les gusta escribir, aportar ideas mediante la producción de un texto visual.

El proceso de investigación-acción permitió ubicar un problema del aula, un hecho real y observarlo; así como reforzar la idea de que es mediante la reflexión sobre la práctica como será posible mejorarla. De ahí, que el proceso realizado permita afirmar que las TIC utilizadas en esta investigación son solo un medio para comunicar, aprender y producir conocimiento. Es importante tener en cuenta que la amplia variedad de recursos y herramientas tecnológicas, así como la renovación permanente de las mismas, harán que en futuros proyectos se utilicen seguramente otras herramientas TIC para obtener los propósitos pedagógicos.

Ahora bien, de acuerdo con los resultados obtenidos y la definición que realiza Dell Hymes (1972) acerca de competencia comunicativa, concluimos lo siguiente:

1. Hymes se refiere a la competencia comunicativa como la capacidad que tienen los hablantes de una lengua de hacer uso de ella, es decir, de cómo ponen en práctica su competencia lingüística (Chomsky, 1957) en situaciones reales de comunicación con hablantes igualmente reales; lo que implica aprender a determinar cuándo hablar, dónde, cómo y cuál es la manera más eficaz de lograr que las contribuciones comunicativas les ayuden a obtener sus objetivos, igualmente comunicativos e ideológicos. En esta perspectiva, la implementación de la unidad didáctica denominada De la clase de literatura a la Red permitió que los alumnos determinaran cómo comunicar un mensaje realizado desde la clase de lengua castellana y que seleccionaran las estrategias más adecuadas para lograr que ese mensaje fuera de calidad, comprendido por los oyentes y estuviera adecuado a las características de YouTube, red a través de la cual fue compartido.

2. Al enseñar a "hacer cosas" con el idioma, nos vemos obligados a determinar de manera clara que en todo proceso de comunicación son im- portantes las reglas de uso de una lengua; las cuales son el resultado de la experiencia social de los integrantes de una comunidad lingüística. El desarrollo de la unidad didáctica De la clase de literatura a la Red incluía estos elementos propios de la competencia comunicativa, lo que le imprimió un nivel de exigencia a los alumnos frente a qué se comunica (el texto) y a quiénes se comunica (usuarios de YouTube, no solo sus compañeros de clase).

3. El uso de herramientas tecnológicas fue llevando a que los estudiantes sintieran la necesidad y evidenciaran la importancia de planear, escribir y reescribir un texto o grabar varias veces haciendo uso de un guion para sus videos. Es decir, en el ejercicio realizado tuvieron en cuenta los elementos fundamentales en la comunicación. Igualmente, los alumnos (quienes comunicaban) tenían como intención clara que su texto no amenazara su imagen negativa y, por el contrario, realzara la positiva; esperando reacciones positivas. Dicho de otra forma, teniendo en cuenta que los alumnos comunicarían de manera pública un texto escrito y producido por ellos en formato audiovisual a través de las redes Facebook y YouTube, al cual puede acceder un número indeterminado de usuarios, ellos, como hablantes, desplegaron un sinnúmero de estrategias para que 1) su imagen negativa no se viera amenazada y 2) su imagen positiva fuera reforzada. Brown y Levinson (citados por Haverkate, 1997), definen esto como el deseo de que los actos de uno sean aprobados, imagen positiva, y el deseo de que uno no vea impedidos sus actos, imagen negativa. Esta consideración pone de manifiesto la necesidad de que el estudiante genere una estrategia comunicativa que le permita reforzar su imagen positiva y, en consecuencia, proteger su imagen negativa.

4. Al proponer la creación de un texto en formato MP4 o texto audiovisual, se les brinda a los alumnos nuevas formas de expresar y comunicar lo que sienten y piensan. 
5. La realización de esta unidad didáctica permitió involucrar diferentes herramientas TIC en el trabajo cotidiano del aula acercándolos a nuevas formas de expresar y comunicar ideas y sentimientos. Esta concepción es coherente con las conclusiones a las que llega la investigación Construcción del conocimiento social a partir del uso de los medios de comunicación para el desarrollo de las competencias comunicativas de los estudiantes (Forero Delgado, Agudelo \& Enciso, 2005).

6. Las unidades didácticas son instrumentos que facilitan la implementación del uso pedagógico de las TIC en el aula y permiten establecer categorías claras de análisis. Esto implica una planeación seria y organizada de las competencias que se pretenden desarrollar, así como de los mecanismos utilizados para tal fin.

7. Al integrar el uso de herramientas TIC al trabajo en el aula de lengua castellana se hace necesario transformar el currículo de manera que se pueda favorecer el enfoque comunicativo en la enseñanza de la lengua.

8. Desde la enseñanza de la lengua castellana, las instituciones educativas pueden brindar a sus alumnos herramientas que aporten a su formación profesional, considerando la competencia comunicativa y el uso de herramientas TIC como promotores de la interacción social en situaciones de comunicación reales y concretas.

9. Las redes sociales representan un potencial pedagógico si se utilizan con objetivos claros y específicos, dado el uso indiscriminado que los jóvenes hacen de estas. En este caso, el uso de Facebook y YouTube como espacios de interacción a través de los cuales, no solo se comparte información, sino mediante los cuales es posible producir conocimiento.

10. La estrategia de evaluación por pares permite fortalecer el aprendizaje cooperativo, en la medida de que, además de fortalecer los aprendizajes de los alumnos, contribuye a aprender entre sí, unos de otros.

\section{Conclusiones y recomendaciones}

Finalmente, con estos resultados podemos recomendar lo siguiente:

Los estudios realizados en torno al tema de competencia comunicativa en la enseñanza del español como lengua propia deben definirse dentro de un enfoque comunicativo y pragmático que no se confunda con habilidades comunicativas (hablar, leer, escribir y escuchar), sino que se complementen.

En este estudio se abordaron algunas herramientas TIC para el fortalecimiento de la competencia comunicativa de los estudiantes; sin embargo, también se utilizaron otras herramientas que facilitaron el proceso de enseñanza y ejecución de la unidad didáctica. Por tal razón, han de considerarse dentro de otra investigación que conduzca a establecer la relación de este uso con la comprensión y/o motivación de los alumnos frente al desarrollo de las unidades didácticas.

Si el uso de las redes sociales e Internet por parte de los alumnos es permanente, la institución educativa puede pensar la posibilidad de implementar estas unidades didácticas en los otros ciclos de la educación (3 y 4 por ejemplo).

En futuras investigaciones relacionadas con tecnologías de la información y la comunicación, TIC, y el desarrollo de la competencia comunicativa se recomienda:

- Disponer de un espacio físico adecuado y acorde con las herramientas TIC utilizadas, teniendo en cuenta que al trabajar con audio y video se requiere de condiciones que garanticen buen sonido e iluminación adecuada.

- Trabajar con grupos cooperativos formales o de base, con el fin de disminuir las dificultades entre los alumnos.

- Contar con observadores externos que realicen el registro fotográfico y videográfico, de modo que se pueda atender de manera eficaz a las dificultades técnicas; o, contar con un ayudante para solventar este tipo de problemas. 


\section{Referencias}

Bertuccelli, M. (1996). ¿Qué es la pragmática? Barcelona: Paidós.

Chomsky, N. (1957). Estructuras sintácticas. México: Siglo XXI.

Escandell, M. (1996). Introducción a la pragmática. Barcelona: Ariel.

Forero, F., Delgado, A., Agudelo, J. \& Enciso, F. (2005). Construcción del conocimiento social a partir del uso de los medios de comunicación para el desarrollo de las competencias comunicativas de los estudiantes. Institución Educativa Distrital El Porvenir. Bogotá: IDEP.
Haverkate, H (1997). La cortesía verbal. Análisis pragmalingüístico. Barcelona: Colección románica.

Hymes, D. (1996). Acerca de la competencia comunicativa. Forma y función, 9. Santafé de Bogotá: Universidad Nacional de Colombia.

\section{Diálogo del conocimiento}

Considero que el tema del artículo es sin duda interesante y pertinente en tanto se orienta a compartir una experiencia de investigación en el aula, de didáctica de la lengua castellana, apoyada en el uso de tecnologías de la información y la comunicación. Sin embargo, el desarrollo del texto es bastante regular, en tanto no se profundiza en la investigación, en las categorías centrales de la misma, en su metodología, sino que se presentan unos resultados y conclusiones que parecen muy generales y no aprovechan la riqueza de la experiencia investigativa.

De hecho, una tesis que proponen los autores es la del paso del uso de TIC a las TAC (como aparece en el título) y de hecho se propone la integración de las redes sociales Facebook y Youtube a la experiencia didáctica, pero luego no se discute este paso de unas tecnologías para la información a las del aprendizaje. En los resultados y conclusiones se plantean cosas muy generales. En términos metodológicos, no se aclara cuántos sujetos participaron, en qué tiempo, qué metodología se utilizó.

De manera más puntual, considero que es necesario que las autoras tengan en cuenta las siguientes consideres para enriquecer su texto investigativo:

- Describir un poco más cómo funcionaron los diferentes aspectos de la pragmática o las reglas de uso de una lengua en el desarrollo de la unidad didáctica "De la clase de literatura a la Red". Se trata de las mismas reglas cuando se usan TIC? cómo se vincula la experiencia social de las y los estudiantes en la misma? ¿Qué elementos de la competencia comunicativa se incluyeron? ¿Cómo fueron los niveles de exigencia? En el texto solo se menciona que allí estuvieron presentes pero no se explica ni cuáles ni cómo.

- En el texto también se dice que los estudiantes utilizaron una "serie de estrategias" para que 1) su imagen negativa no se vea amenazada y 2) su imagen positiva sea reforzada. ¿Cuáles estrategias?

- En el texto se argumenta que es necesario cambiar el currículo para que funcionen unidades didácticas como la desarrollada en el estudio, qué cambios se hicieron en el currículo, ¿o cuáles sugieren?

-Sería importante mencionar cuáles fueron los mecanismos de producción de conocimiento en Facebook, ¿cómo se manifestaron o produjeron, porqué allí efectivamente se habla de producción de conocimiento y no de información?

Rocío Rueda Ortiz 